\title{
LA PLENITUDO POTESTATIS E LA VENERATIO IMPERATORIS COME PRINCIPIO DOGMATICO DELLA POLITICA DELLA TARDA ANTICHITÀ. UN ESEMPIO CHIARO: LA NOTITIA DIGNITATUM $\mathrm{Di}$ CONCEPCIÓN NEIRA FALEIRO*
}

Scopo di questo articolo è dimostrare l'uso della iconografia nella Tarda Antichità per legittimare una solida gerarchia politica, che si riflette nella Notitia Dignitatum. Una iconografia d'aspetto politico ed allegorico che mostra un panorama storico-sociale, base per la sistemazione, combinazione e struttura degli elementi dell'icona. La legittimità dell'ordo dignitatum e la potestas et ueneratio imperatoris, si uniscono giuridicamente alla Chiesa sotto il dominium imperatoris attraverso un parallelismo dei reciproci poteri. Questo è il significato allegorico dell'immagine al servizio del simbolismo testuale, arte per l'arte, la iconografia fondata sul testo, la tecnica greca della $\tau \varepsilon \chi v o \pi \alpha i ́ r v i \alpha o v$ e il suo risultato $\tau \varepsilon \chi v o \pi \alpha i ́ r v i o v$.

\section{Introduzione}

La Notitia Dignitatum omnium tam ciuilium quam militarium in partibus Orientis et Occidentis $-N D$ - è un documento militare della Tarda Antichità IV-V sec.- che elenca meticolosamente, sotto l'aspetto civile e burocratico, la distribuzione militare in tutto l'Impero romano, ed offre una informazione complessiva sullo stato dell'Impero e, quindi, sulla situazione militare, che doveva essere preoccupazione preminente nei periodi di crisi. Si tratta di un archivio di Stato aggiornato periodicamente, una sorta di annuario dell'Impero: ogni modifica, civile oppure militare, accaduta nell'Impero era registrata in questo tipo di catalogo per la conoscenza dell'imperatore. Il termine stesso di Notitia si riferisce a registri completi di ciò di cui si parla, a

\footnotetext{
- Vorrei ringraziare il Prof. dr. Lukas de Blois per l'invito a questo Convegno Internazionale, che mi ha permesso di ampliare ulteriormente una ricerca iniziata ad Oxford grazie ad una borsa di studio post-dottorato di insegnamento e ricerca scientifica (EX99 50443535G) concessa dal Ministerio della Pubblica Istruzione Spagnolo nel Regno Unito per un periodo di due anni. Le discussioni scientifiche avute con il Prof. dr. Frgus Millar con cui ho lavorato in questi due anni-, il Dr. Walter Pohl di Vienna, il Dr. László Borhy di Budapest e il Prof. dr. Michael D. Reeves di Cambridge lo hanno arricchito. Vorrei anche ringraziareil Dr. Antonio Polichetti per le correzioni fatte al mio italiano nelle bozze di questa redazione. La posta elettronica dell'autrice è: notitia@mexico.com.
} 
differenza delle liste dei concili ecumenici, dei canoni conciliari, delle lettere dei sinodi o di altri documenti simili. ${ }^{1}$

Il documento si apre con un index che classifica, in maniera gerarchica, le dignitates tam ciuilium quam militarium del periodo - uir illustris, spectabilis et clarissimus -; seguono i capitoli che, attraverso una ricca e curata iconografia sviluppano nel testo l'elemento iconografico; ogni capitolo si chiude con la descrizione dell'officium.

\section{La potestas imperatoris: aspetti iconografici della politica nella Tarda Antichità}

La imago imperatoris è la rappresentazione che unisce le due sezioni del documento corrispondente alle due parti dell'Impero; nella metà precisa della $N D$ ci sono due imagines clipeatae ${ }^{2}$ - Diuina Prouidentia et Diuina Electio - sviluppate conformemente ai precetti iconografici e simbolici bisantini: l'imperator, considerato come dominus, è al centro dell'universo, da cui tutto e tutti discendono, è l'incaricato di un imperium infinitum stabile e coeso, realizzato all' insegna di un ordo dignitatum costituito da se stesso ${ }^{3}$; cosí, l'imago clipeata, l'unica figura del documento senza testo, conferma il ruolo politico dell'imperatore, che è sviluppato attraverso le coordinate iconografiche delle virtù di un capo di Stato ${ }^{4}$. Le due immagini riflettono una ideologia politica sostenuta, l'ordo dignitatum imperii, del quale codicilli, epistulae et Liber Mandatorum - CTh. 6.22.5 - raccoglievano ed esponevano

' G. Panciroli, N.utraque D. cum Orientis tum Occidentis ultra Arcadii Honirique tempora. Et in eam G. Panciroli commentarium. Item de magistratibus municipalibus eiusdem auctoris liber (Venezia $1593), 2^{\vee}$ s.: “....omnia in officio facilius continenda, cuncta in libro descripta, habuerunt, qui prius Breuiarium, seu ratiocinarium imperii, mox Notitia est appellatus, in quo ciuiles magistratus, et militares, legiones, auxilia, cohortes, uexillationes singulae cum suis symbolis, et insignibus officiisque positae comprehendebantur..."

${ }^{2}$ R. Winkes, Clipeata Imago (Bonn 1969). C. Nordenfalk, Der Kalender vom Jahre 354 und die lateinische Buchmalerei des IV Jahrhunderts (Göteborg 1936); idem, Studies in the History of book illumination (Londra 1992); M. Schapiro, 'The Carolingian Copy of the Calendar of 354', Art Bulletin 22 (1940), 270-272; A.W. Byvanck, 'Antike Buchmalerei III. Der Kalender vom Jahre 354 und die ND', Mnemosyne ser.3 (1940), 177-198. H. Stern, Le Calendrier de 354; Étude sur son texte et sur ses illustrations (Parigi 1953). M. Renee Salzman, On Roman Time. The Codex-Calendar of 354 and the Rhythms of Urban Life in Late Antiquity (California 1990).

${ }^{3}$ H.P. L'Orange, Apotheosis in Ancient Portraiture (Oslo 1947), 122-124. D. Mertens, 'Bisanzio', Il pensiero politico Medievale (Bologna 1999), 9-17.

${ }^{4}$ J.R. Fears, Princeps a Diis Electus: The Divine Election of the emperor as a Political Concept at Rome, Papers and Monographs of the American Academy in Rome 26 (Roma 1977, recensione da P.A. Brunt, Journal of Roman Studies 69 [1979], 168-175). Cl.M.M. Bayer, "Essai sur la disposition des inscriptions par rapport à l'image. Proposition d'une typologie basée sur des pièces de l'orfèvrerie rhéno-mosana", Épigraphie et iconographie. Actes du Colloque tenu à Poitiers les 5-8 Octobre 1995, Centre d'Études Supérieures de Civilisation Médiévale, cur. R. Favreau, (Poitiers 1996), 1-25. 
le cariche e le responsabilità della dignitas, regolarizzando la presa di possesso dell'incarico attraverso un atto pubblico presieduto dall'imperatore - CTh. 6.22 .8 -.

La rappresentazione iconografica conservata nella tradizione manoscritta del documento è costituita dalle immagini di Diuina Prouidentia et Diuina Electio. ${ }^{5} \mathrm{Da}$ un punto di vista metaforico questo frontespizio rappresenta, da una parte, l'imperatore come la persona cui è consacrata l'opera, dall'altra, il garante di una organizzazione gerarchica e burocratica suggerita dalla divinità che, a sua volta, ha nominato l'imperator come responsabile di questa struttura di governo. In questo modo, la $N D$ fu concepita come documento di propaganda politica per le due parti dell'Impero, e come apologia della politica teodosiana: ${ }^{6}$ il linguaggio iconografico e pittorico corrisponde ad una sistemazione ideologica più che alla realtà storica del periodo. Il libro, nel documentare il distacco "fisico" dell'immagine dal testo, rivela in maniera esemplare il funzionamento come comunicazione autonoma rispetto a quella scritta, come testo a sua volta. ${ }^{7}$

Le imagines clipeatae, rappresentanti massime di una simbologia cosmica, determinano e circoscrivono ambedue le immagini, attribuendo un indiscutibile valore di universalità, rispetto ai quali guardano l'immagine; paradossalmente la risposta è data dal medesimo concetto di sottomissione che rafforza l'istituzione; si tratta di una retorica e di una liturgia che si usa per dimostrare, da una parte, il primato e la pluralità della potestas imperatoris - Diuina Prouidentia -, dall'altra, la temporaneità e caducità della stessa potestas -Diuina Electio.$-^{8}$ La Prouidentia, facoltà di prevedere

\footnotetext{
${ }^{5}$ C. Neira Faleiro, La ND: nueva edición critica y comentario histórico, Tesi di Dottorato, Pubblicazioni della Università Complutense de Madrid (Madrid 2002, in stampa); idem, 'Ante una nueva edición crítica de la ND', Actas del II Congreso Hispánico de Latin Medieval 2 (1998), 697-707; idem, 'Nuove prospettive dei Manoscritti Trentini della ND', Studi Trentini di Scienze Storiche 80.1.4 (2001), 823-833; idem, 'El Parisinus latinus 9661 y la ND: nuevas perspectivas', Latomus (in stampa).

${ }^{6}$ S. McCormack, 'Latin Prose Panegyrics', in T.A. Dorey, ed., Empire and Aftermath (Londra 1975), 143-205 = Revue des études augustiniennes 22 (1976), 29-77; C.E.V. Nixon, 'Constantinus Oriens Imperator: Propaganda and Panegyric. On reading Panegyric 7(307)', Historia 42 (1993), 229-246.

${ }^{7}$ P.G. Berger, The Insignia of the ND: A contribution to the study of Late Antique Illustrated Manuscripts (New York 1981), 140-141 n.318; A. Schön, 'Il silenzio del mito: osservazioni psicoanalitiche', in M. Grazia Ciani, cur., Le regioni del silenzio: studi sui disagi della comunicazione (Padova 1983), 15-25; F. Minissale, 'Il colore e le immagini del colore (colore e linguaggio nelle Georgiche di Virgilio)', in La parola delle immagini e delle forme di scrittura. Modi e tecniche della comunicazione del mondo antico, Dipartimento di Scienze dell'Antichità dell'Università degli Studi di Messina 1998 (Messina 1998), 205-227.

${ }^{8}$ A.B. Van Houten, 'The influence of syntax on visual perception', Anthropological Linguistics 19 (1977), 355-358; M. Cagiano de Azevdo, 'Il colore nella antichitá', Aevum 28 (1954), 151-167; V.W. Turner, The ritual process; structure and antistructure (Londra 1969), spec. c.5; H.C. Conklin,
} 
il futuro, fu considerata dalla mentalità romana effluvio divino - Plinius, Panegyricus 10.4 - nel I secolo d.C. La prouidentia era un aspetto della divinità dell'imperatore -CIL.6.1.2028, $\mathrm{d} ; 5.1871-$, fino al regno di Constantino, diventando attributo di Dio. ${ }^{9}$

Il clipeus, come immagine rotonda, rappresenta la totalità cosmica dell'infinito rispetto al suo incessante e continuo movimento, il fondamento centripeto che stabilisce e determina le regole nel suo aspetto terreno e divino. Nel mondo greco, soprattutto con Platone ed Aristotele c'era il convincimento di una profonda corrispondenza fra la disposizione del cosmo, l'organizzazione della società e l'organizzazione dell'individuo stesso come partecipe di quella società. L'obiettivo fondamentale della politica era ottenere che l'organizzazione della società si adeguasse all'ordinamento cosmico e che l'individuo si avvicinasse all'essenza di cui è immagine e portatore nella politica.

Da questi principi, nella seconda metà del IV secolo, la liturgia cristiana concepì l'immagine di Cristo come fondamento di tutto quello che significa e rappresenta l'essenza umana; in questa struttura tonda, ${ }^{10}$ l'icono arriva alla stessa maniera e con lo stesso senso a tutti i livelli socio-culturali della collettività, senza essere capita o interpretata male; così, lo scopo della espressione iconica si rivela incontestabile, di facile e chiaro discernimento, nel contesto ufficiale della medesima comunità che lo ha concepito e sviluppato, escludendo la possibilità di conferire concetti diversi oppure opposti tra loro, ad una rappresentazione iconografica: "il colore è per sua natura dotato di un potere assai ampio, che va dalla semplice sollecitazione sensibile... alla capacità evocativa e al simbolismo più raffinato... Basta ricordare l'importante ruolo tenuto dal colore in tutte le antiche cosmogonie, e i suoi riflessi sul costume e nella vita sociale". ${ }^{11}$

'Colour Categorisation' American Anthropologist 75 (August 1973), 931-942; M.H. Bornstein, 'The influence of visual perception on culture', American Anthropologist 77 (1975), 774-798; B. Brent \& P. Kay, Basic Color Terms: their universality and Evolution (Berkeley 1969), 16-17: “...three appears to be a positive correlation between general culture complexity (and/or level of technological development) and complexity of color vocabulary...". Per il significato del colore nella società greca vid. Plato, Meno 74-76; Aristotele, De Sensu 439a-440b; De Coloribus 791a-799b.

${ }^{9}$ A. Wallace-Hadrill, 'The Emperor and his Virtues', Historia 30 (1981), 298-323.

${ }^{10}$ H.P. L'Orange 1947, op.cit. (n.3), 124-125. E. Beurlier, Essai sur le culte rendu dux empereurs romains (Parigi 1890); idem, Le culte impérial, son histoire et son organisation depuis Auguste jusqu'à Justinien (Parigi 1891); A. Wierzbicka, 'The meaning of color terms: cromatology and culture', Cognitive Linguistics 1.1 (1990), 99-150.

${ }^{11}$ R. d'Avino, 'La visione del colore nella terminologia greca', Ricerche Linguistiche 4 (1958), 99134, ad 129-130 n. 130; C. Mangio, 'Cenni sulle teorie cromatiche dei greci e loro applicazione architettonica', Studi Classici e Orientali 10 (1961), 214-223; M. Caccamo Caltabiano, 'Immagini/parola, grammatica e sintassi di un lessico monetale', La parola delle immagini e delle 
Parallelamente all'interpretazione sacra dei clipea, questa struttura tonda fu accettata per rappresentare l'aspetto politico del potere, come è esposto nella $N D$ : il busto frontale dell'imperatore con l'aureola è il modo universale per indicare le connotazioni divine, ${ }^{12}$ la glorificazione mondana della maiestas imperatoris in forma antropomorfa, la deferenza e il rispetto alla potestas che procede da Dio attraverso l'imperator, secondo la concezione ierocratica del potere; l'aureola segna la deferenza di una persona, mentre lo sviluppo della composizione frontale e simmetrica e la posizione frontale dell'imperatore viene considerata come espressione di potere e glorificazione della imago. La presenza dei due genii alati che sostengono il clipeus fanno riferimento alla apoteosi divina dell'imperatore, considerato come Dio onnipotente. ${ }^{13}$ Ogni atteggiamento del dominus è espresso in un modo premeditato per definire la sua posizione centrale nello Stato, il rector totius orbis e la sua natura soprannaturale. In un'unica struttura tonda, il clipeus, è riassunto il concetto di armonia e sintesi politica, sviluppata nel documento come immagine, non soltanto tra i diversi livelli sociali, ma anche sotto l'aspetto politico e religioso della società; l'equilibrio tra questi livelli è necessario per l'articolazione dello Stato. L'imperatore è il punto fisso, il vero centro immobile ed infinito intorno al quale gravita ogni cosa, progettato secondo una chiara liturgia celeste.

L'idea di Stato deve essere concepita ed associata ad un potere che si trova più in alto della volontà individuale; è un potere universalmente riconosciuto ed accettato, esercitato secondo norme o regole conosciute o conoscibili. Il riconoscimento e l'accettazione di questo potere esercitato secondo determinate regole implica il riconoscimento e l'accettazione

forme di scrittura. Modi e tecniche della comunicazione del mondo antico, Dipartimento di Scienze dell'Antichità dell'Università degli Studi di Messina 1998 (Messina 1998), 57-74.

${ }^{12}$ P. Skubiszewski, 'Le titre de "Roi de gloire" et les images du Christ: un concept théologique, l'iconographie et les inscriptions', in R. Favreau, cur., Civilisation Médiévale II. Épigraphie et iconographie. Actes du colloque tenu à Poitiers les 5-8 octobre 1995, Centre d'Études Supérieures de Civilisation Médiévale (Poitiers 1996), 229-258; D. Scortecci, 'Il medico, l'empio, l'imperatore e il santo: dal ritratto nimbato alla sacra aureola', in A. Isola, E. Menestò \& A. Di Pilla, curr., Curiositas, Studi di Cultura Classica e Medievale in onore di Ubaldo Pizzani (Napoli 2002), 589-608; Libellus de cerimoniis aule imperialis, c.19, cur. Schramm, Kaiser, Könige und Päpste 3.344: "Imperator ferat camisum, ex subtilissimo et candidissimo bisso contextum, cum aurea bulla, ornatum a pedibus ad mensuram brachii in circuitu de auro frigio" (la citazione è stata presa da A. Paravicini Bagliani, $I l$ corpo del Papa [Torino 1994], 52-53).

${ }^{13}$ L'esempio trasmesso nella $N D$ è il più antico delle vittorie con lo sguardo rivolto frontalmente che sostengono il busto dell'imperatore; le vittorie alate che portano la imago imperatoris appaiono successivamente nei dittici consolari intorno al 480. R. Grigg, 'Symphônian Aeidô tês Basileias: An Image of Imperial Harmony on the Base of the Column of Arcadius', Art Bulletin 49 (1977), 469-482. 
dell'obbligo di sottostare alle regole stesse. Queste caratteristiche generali fanno dello Stato un'istituzione. D'altra parte l'idea di Stato viene associata al concetto di potere e al rispetto di norme che regolano i modelli di comportamento per quanti si riconoscono nell'istituzione stessa, o comunque di comportamenti compatibili con l'istituzione, che ne possano garantire l'esistenza e la sopravvivenza. Questa forza normativa, ragione d'essere dello Stato stesso, introduce necessariamente la nozione di diritto, di legge. La concezione dello Stato in termini di diritto significa riconoscere il potere dello Stato che si esercita in nome della legge superando la mera esistenza fattuale del rapporto di potere/obbedienza. La concezione augustea di compimento politico prendeva come principio l'armonia fra le diverse entità che formavano il concetto di Stato, cioè, il Senato e il popolo, per i quali il princeps agisce.

Alla stessa maniera, la struttura del clipeus è usata non soltanto per sottolineare l'atteggiamento divino dell'imperatore al centro dell'icona, ma anche per evidenziare l'atteggiamento divino dell'imperatore come organismo per designare i suoi rappresentanti diretti mediante la sua sapienza divina, concessa soltanto all'imperatore; così, si può capire la presenza delle uirtutes imperatoris nei clipea - piano antropomofo, Diuina Prouidentia - e la presenza delle stagioni dell'anno - piano divino, Diuina Electio -

\section{Canonizzazione delle virtù di un bravo capo di Stato}

Gli attributi rappresentati nei clipea della Diuina Prouidentia - uirtus, scientia rei militaris, auctoritas, felicitas - erano virtù attribuite all'imperatore dall'età di Cicerone De Imperio Cnei Pompei $10 .{ }^{14}$ La uirtus era un elemento della divinità - Sallustio, Catilinariae 1.4; 20.2 -; secondo Cicerone De Legibus 2.8.19 questa uirtus indicava prerogative di temperamento militare: un bravo militare doveva riunire in sè forza, sagacia mentale e fisica, coraggio, perseveranza - Cicerone, De Natura Deorum 2.31.79; Giovenale, Satirae 1.115 -. Nella tarda antichità la uirtus divenne un attributo umano.

La scientia rei militaris - Caesar, Bellum Africanum 3.23, 7.57 - era la conoscenza della disciplina militare, secondo il concetto di uirtus dal punto di vista bellico; così, uirtus et scientia rei militaris si riferiscono agli obblighi militari dell'imperator. L'equilibrio e la congiunzione dell'aspetto civile e militare dello Stato si riuniscono nel massimo rappresentante del

\footnotetext{
${ }^{14}$ Cicerone, Pro lege Manilia 28; Ammiano Marcellino, Panegirico a Giuliano 25.4.1ss; J.A. Crook, 'Some remarks on the Augustean Constitution', Classical Review 3 (1953), 10-12; J. Hellegouarch, Le vocabulaire latin des relations et des partis politiques sous la République (Parigi 1963).
} 
popolo: due aspetti che, dal punto di vista della costituzione, sono indipendenti, però, giuridicamente, dipendono della persona imperatoris. ${ }^{15}$

La felicitas si riferisce al concetto di fecondità dello Stato - Plinius, Historia Naturalis 7.43; Plinius Minor, Epistulae 3.19.6-.

La auctoritas era un concetto fissato con forza nella società romana già nel periodo repubblicano, era l'accettazione esplicita e legale di un nuovo rex accettato dal popolo; tale assenso era dato dai patres conscripti. L'elezione della persona nominata si fondava sulla considerazione e celebrità della medesima davanti a tutti, ottenuta grazie alle sperimentate capacità militari, il cui risultato finale consiste nella conquista della prospettiva civile dell'Impero e nell'estrinsecarsi delle qualità militari innate - Cicerone, Pro Lege Manilia 28 -

La felicitas e l'auctoritas corrispondono all'aspetto politico dello Stato. La prosperità e il benessere dell'Imperium si acquistano mediante la formulazione di decreti e leggi da parte dello stesso imperatore, leggi che si applicano all'Impero romano, anche nella scelta dei suoi rappresentanti diretti per eseguire i mandata imperatoris. Il riassunto di questi fu il Liber Mandatorum ${ }^{16}$ che assegnava il giorno del conferimento dell'incarico ad ogni governatore di provincia da parte dell'imperatore. Il contenuto del Liber erano le leggi dell'Impero - senatus consulta, constitutiones imperatoris, plebisciti - e iussa di carattere locale; alcuni erano del periodo precedente alla invasione romana, altri furono scritti dagli stessi amministratori o giuristi provinciali - CTh. $6.29 .10-.^{17} \mathrm{Il}$ suo valore risiedeva in una raccolta degli iussa imperatoris per le provincie e nella manifestazione della potestas dell'imperatore sull'Impero, che trasmetteva la sua auctoritas ai suoi rappresentanti immediati nelle provincie; il Liber Mandatorum è il simbolo del potere imperiale in una realtà legislativa romana lontana dalla cancelleria

\footnotetext{
${ }^{15}$ Il termine costituzionalmente deve essere riferito ad un punto di vista giuridico quale decreto, ordine, editto.

${ }^{16}$ L'unica fonte storica che parla, iconograficamente, di un Liber Mandatorum è la ND, Not.Occ.44. O. Karlowa, Römische Rechtsgeschichte (Leipzig 1885), 869. Cf V. Marotta, Mandata Principum (Torino 1991). Il codex portava sulla copertina frontale una legenda di riconoscimento pubblico e saluto riportata all'ufficiale annuale della carica: FL INTALL COMORD PR (=FLOREAS (oppure FELICITER)/INTER/ALLECTOS/COMITES/ORDINIS/PRIMI).

${ }^{17}$ A. dell'Oro, 'Mandata' e 'litterae'. Contributo allo studio degli atti giuridici del 'princeps' (Bologna 1960), spec. 74-76; J.-P. Coriat, 'Du pouvoir dans l'antiquité: mots et réalités', in C. Nicolet, cur., Technique législative et systéme de gouvernement à la fin du Principat: la romanitè de l'Etat moderne (Ginevra 1990), 121-238. G. Coppola, Cultura e potere. Il lavoro intelletuale nel mondo romano (Milano 1994). C. Neira Faleiro, 'Documentos de Estado en el Bajo Imperio; un caso particular: la $N D^{\prime}$, in S.Crespo Ortiz de Zárate \& A. Alonso Ávila, curr., Scripta Antiqua in Honorem A.Montenegro Duque et J.M.a.Blázquez Martinez, Magistris Optimis (Valladolid 2002), 761-775.
} 
di Stato, definita da una centralizzazione normativa che esigeva una specializzazione del sistema giuridico e poneva “...le premesse per quella combinazione fra burocrazia e legislazione, fra diritti dei sudditi e arbitrio del principe (e dei suoi funzionari) che costituisce tanta parte dell'ultima storia imperiale..." (56 f.)-. ${ }^{18}$

I due aspetti per applicare la potestas, antropomorfo e divino - Diuina Prouidentia et Diuina Electio ${ }^{19}$ - sono sviluppati rispettivamente da un punto di vista simbolico e in maniera indipendente. La complementarietà lessicale di entrambe le immagini senza testo si capisce dalla concezione e dallo scopo dello stesso folium: divenuta autonoma, l'immagine assume la funzione propria del testo, quella di produrre un discorso, un discorso visuale. ${ }^{20}$ Quella è la vera natura del potere politico, l'autonomia del potere civile, la cui finalità fondamentale è difendere la giustizia attraverso le leggi concepite dalla divinità e concesse all'imperatore. Così, il concetto di proprietà è associato con quello di potere o autorità in quanto il possesso di beni materiali e la capacità di esercitare qualche potere sono due aspetti inseparabili di una medesima potestas. ${ }^{21}$ La prima illustrazione - Diuina Prouidentia - definisce le virtù teologali dell'imperatore, ogni attività del governo del sovrano Vegetius, De Rebus Bellicis, praef. 5; 21.2 - ; la seconda illustrazione -Diuina Electio- è l'elezione divina dell'imperatore, il riconoscimento e l'accoglienza della sua maiestas da tutti; Dio è considerato come l'unica fonte di legittimità del potere pubblico e dell'aspetto giuridico. Egli garantisce e salvaguarda il principio dell'origine divina del potere, per attribuire alla ragione dell'uomo la facoltà di individuare l'istituzione della potestas ciuilis, che permette lo svolgimento della convivenza. L'istituzione

\footnotetext{
${ }^{18} \mathrm{~F}$. Grelle, 'Le categorie dell' amministrazione tardoantica: officia, munera, honores', Società romana e impero tardoantico (Roma 1986), 37-57, spec. 56-57; idem, 'L'organizzazione amministrativa', in Storia di Roma: L'età tardoantica: crisi e trasformazione IIV/1 (Torino 1993), 77-82; C. Vismara, Le provincie dell 'Impero, Il funzionamento dell 'Impero (Roma 1989); P.S. Barnwell, Emperor, Prefects and Kings. The Roman West 395-565 (Chapel Hill, NC 1993); A. Schiavone, 'Il mondo tardoantico', in Storia Medievale (Roma 1999), 43-64.

${ }^{19}$ G. Downey, Themistii Orationes quae supersunt (Leipzig 1965), Oratio 15. E.S. Ramage, The Nature and Purpose of Augustus' Res Gestae (Stoccarda 1987), spec. 73-100.

${ }^{20}$ E. Kitzinger, 'The Role of Miniature Painting in Mural Decoration', in K. Weitzmann, W.C. Loerke, E. Kitzinger \& H. Buchthal, curr., The Place of Book Illumination in Byzantine Art (Princeton 1975), 99-142. Not.Or.3 e Not.Occ. 2 prendono come base i principi di prospettiva tridimensionale che con l'abbozzo dell'idea di profondità conseguono lo scopo della illusione spaziale. La grande misura delle personificazioni fa pensare ai modelli originari delle pitture parietali del quarto secolo, copiati nei codices e che hanno perduto la funzione originaria, ovvero essere guardate da un pubblico con una chiara funzione decorativa.

${ }^{21}$ H. Löehken, Ordines Dignitatum. Untersuchungen zur formalen Konstituierung des spätantiken Führungsschicht (Köln 1982), spec.c.3, 848-868.
} 
dell'autorità deriva da Dio, poichè egli ha concesso la facoltà di istituire una forma di autorità per il bene della società che l'avesse richiesta. Il potere dell'imperatore è riconosciuto anche attraverso la rappresentazione delle quattro stagioni: inviolabilità e perennità della potestas che simboleggia mentre "il silenzio diviene imposizione quando uno dei due riconosce il prestigio o la supremazia dell'altro; scelta e imposizione possono esprimere rispettivamente affermazione e riconoscimento di una leadership... Il silenzio sottolinea il riconoscimento di un potere con il quale non si può competere" (33-4 ff.). ${ }^{22}$

\section{Potestas imperatoris in absentia: riconoscimento e accoglienza pubblica}

La persona incaricata di queste compilazioni, con attribuzioni amministrative e giuridiche, era il primicerius notariorum, con il compito di compilare le tabullae honorum che raccoglievano gli incarichi degli impiegati dell'Impero nei codicilli; questi raggruppavano gli obblighi annuali dello Stato, compresa la retribuzione della dignitas più importante, il praefectus praetorio. La compilazione di questi mandata era fatta nel laterculum maius, ${ }^{23}$ un codex purpureo con nastri dello stesso colore, che rappresentava il carattere ufficiale del documento risultato dell'unione di pergamene precedenti:

"Altre qualità che rendevano unica la porpora erano la stabilità e
l'inalterabilità... Uso rituale e cerimoniale del rosso... funzione
apotropaica... accrescimento della forza vitale..." (114 f.); "La
porpora segnala... l'intoccabilità, la proibizione di attendere
all'integrità fisica di quella persona che porta questo colore. Dal
secolo IV... la porpora s'imporrà come segno distintivo ed
esclusivo della dignità imperiale, gelosamente custodita da
un'ampia legislazione suntuaria restrittiva....24

\footnotetext{
${ }^{22} \mathrm{P}$. Scarpi, 'L'eloquenza del silenzio; aspetti di un potere senza parole', in M. Grazia Ciani, cur., Le regioni del silenzio: studi sui disagi della comunicazione (Padua 1983), 31-41; $\mathrm{M}^{\mathbf{3}}$ Dolores Caínzos, 'Aeternitas o desaparición de Roma? Dos visiones de la sociedad romana', Quaderni urbinati di cultura classica 63.3 (1999), 139-161.

${ }^{23}$ E. Böcking, $N D$ et administrationum omnium tam ciuilium quam militarium in partibus Orientis et Occidentis (Bonn 1839-1853), vol.II, 407-409; G. Bonolis, I titoli di nobiltà nell'Italia Bizantina (Firenze 1905), 74-75; E. Posner, Archives in the Ancient World (Cambridge, Massachusetts 1972), 207-208; Berger 1981, op.cit. (n.7), 25-37, glossario, s.u.Codicilli, 175-183; R. Grigg, 'Portraitbearing Codicils in the illustrations of the ND', Journal of Roman Studies 69 (1979), 107-124.

${ }^{24}$ A. Casartelli, 'La funzione distintiva del colore nell'abbigliamento romano della prima etá imperiale', Aevum 1.72 (1998), 109-125, ad 115-116; B.M. Metzger, 'When did scribes begin to use writing desks?', in Akten des XI.Internaternationalen Byzantinisten-Kongresses (Monaco di Baviera 1960), 355-362; R. Delbrück, 'Der spätantike Kaiserornat', Die Antike 8 (1932), 7-15; O. Treitinger,
} 
Le fenditure sulla copertina del codex erano per i fermagli di metallo destinati a proteggerlo - Not.Or.18, Not.Occ.16-. Assegnare questi documenti il nel giorno della investitura della carica corrispondente definiva il rapporto fra la dignitas e il carattere sacro imperatoris, anche negli atti ufficiali fatti dai suoi delegati: il ritratto dell'imperatore sui codicilli era la promulgazione del potere reale e simboleggiava la sua praesentia "...misleading to speak of an administrative hierarchy. The pyramid of emperor, praetorian prefects, vicars and provincial goverments looks very neat as set out in the $N D$, but there was in reality no rigid chain of command...". 25

L'officium del magister scriniorum - Not.Or.19, Not.Occ.17- era composto dal:

1) magister memoriae, che compilava e faceva conoscere le sentenze dell'imperatore di carattere privato oppure di contenuto internazionale, le adnotationes, a guisa di glossa sui margini dei documenti della amministrazione con l'accettazione autografa dell'imperatore; le consegne dovevano essere fatte in un libellus; il magister memoriae, quindi rappresenta il ponte fra l'imperatore e il populus; ${ }^{26}$

2) magister epistolarum, il quale compilava le risposte consegnate dall'imperatore alle delegazioni straniere e ai delegati di provincia, le legationes ciuitatum, ${ }^{27}$ esaminava e controllava i consigli giudiziari,

\footnotetext{
Die oströmische Kaiser- und Reichsidee nach ihrer Gestaltung im höfischen Zeremoniell : Vom oströmischen Staats- und Reichsgedanken (Darmstadt 1956), 9-10. Plinius Maior, Historia Naturalis 9.136; Macrobius 1.6.7.

${ }^{25}$ A.H.M. Jones, The Later Roman Empire (Oxford 1986), 374-375. Cf. E. Palazzo, 'Tituli et enluminures dans le haut Moyen âge (IX-XIe siècles): fonctions liturgiques et spirituelles', in R. Favreau, cur., Épigraphie et iconographie. Actes du Colloque tenu à Poitiers les 5-8 Octobre 1995, Centre d'Études Supérieures de Civilisation Médiévale (Poitiers 1996), 167-191.

${ }^{26}$ Böcking 1839-1853, op.cit. (n.23), vol.II, 414-416 “...imperatoribus in memoriam reuocaret, sed quod acta et decreta memoriae ac futurae obseruantiae causa asseruaret. Immo, memoria, ipsa letteras, documenta instrumentaue, acta gestaque memoriae conseruationisue caussa reposita significat... genus imperialis scripturae, oppositum rescriptis uel epistolis, pragmaticis sanctionibus ceterisque legius quas quaestor dictabat, in caussis gratiae, administrationis, politicae financiarumque quae hodie dicimus, sed et in caussis ad iuris dictionem spectantibus emittebatur..."; 325-327 n.4: “...Leges dictandae: ...'dictare'... non satis accuratam esse censeo: dictare in his locis est quasi schedam conscribere, scriptionem qualemcumque primum delineare... siue ipse quis scribat siue calamo alterum praedicta excipere iubeat; ita dictata postquam principis manu, ubi opus erat, approbata signataque erant, rescribebantur, in mundum redigebantur, expediebantur, ut hodie dicimus..."

${ }^{27}$ Böcking 1839-1853, op.cit., (n.23) vol.II, 417-418: "Legationes exterarum gentium magister officiorum prouincialium, ciuitatium siue corporum praefectus praetorio excipiebat, sed ubi imperialis
} 
le consultationes, fatte all'imperatore dagli impiegati della amministrazione centrale;

3) magister libellorum, che organizzava le procedure giuridiche, cognitiones, sorvegliate direttamente dall'imperatore senza precedente scritto, ed esaminava le richieste, preces, fatte all'imperatore; i documenti di questo dipartimento erano compilati in codici di legno, per essere rivisti e corretti dal quaestor; il risultato era un abbozzo scritto su papiro e trasmesso direttamente all'imperatore;

4) magister epistolarum graecarum, si occupava delle lettere e dei documenti scritti in greco, oppure traduceva in greco i documenti scritti in latino, egli è attestato solo in Oriente. ${ }^{28}$

La presenza dell'imperatore era necessaria per legittimare e legalizzare ogni atto ufficiale, giuridico o amministrativo fatto nelle provincie dell'Impero ...per ipsum enim omnia, quae quidem sic data sunt per Moysen, ut umbra essent, uel figura futurorum, ut apparente ueritate, figura cessaret. Sicut enim absente imperatore, imago eius habet auctoritatem, praesente non habet; ita et haec ante aduentum Domini tempore suo obseruanda fuerunt, praesente autem, carent auctoritate. Numquid aliquis uicem domini agens, ipso praesente, dominatur? Si praefectorum uicarii, praesentibus eis priuati sunt; quanto magis serui, praesente domino, etiam ipsi in obsequio debent uideri (PL XVII coll.431-432) -. ${ }^{29}$ L'imperatore, dinanzi alla impossibilità di una presenza fisica, in questi atti si rivolgeva alla presenza iconica della sua immagine: il valore di questa immagine e le conseguenze sul governo erano

scientia consulenda erat, i.e.quotiens petitiones non statim lectae pensataeque reiciendae uidebantur, principi transmissae ab ipso post adhibitum consistorium decidebantur, recitationes autem, consultationes supra dictorum uirorum illustrium aliarumue dignitatum, ad quas legationes uenerant, ac prece petitionesque legatorum tractabat scrinium epistolarum sub suo magistro: in caussis itaque legationum hi scriniarii secundum nostrum loquendi usum principis referendarii, archiuarii atque correspondentes erant."

${ }^{28}$ B. Hemmerdinger, 'Les lettres latines a Constantinople jusqu'à Justinien', Byzantinischen Forschungen 1 (1966), 174-178; G. Dagron, 'Aux origines de la civilisation byzantine: langue de culture et langue d'État', Revue historique 241 (1969), 36-46.

${ }^{29}$ Ambrosianus, Commentaria in Epistolam B.Pauli ad Colossenses II, ad uers. 16-17, PL, XVII.431433. H. Kruse, Studien zur offiziellen Geltung des Kaiserbildes im römischen Reiche (Paderborn 1934), 79-81 e 101-103. D. Mertens, 'Responsabilità temporale della Chiesa e idea papale del primato', in Il pensiero politico Medievale (Bologna 1999), 71-83. Severiano di Gabala, De mundi creatione Or.6.5 nel quinto secolo, scriveva che il ritratto del sovrano doveva essere collocato nei tribunali e in ogni altro luogo ove si esercitasserero funzioni pubbliche: soltanto in tal modo una sentenza emanata da un funzionario dell'imperatore poteva aver valore; su questa prassi e i suoi precedenti, che ritornano al secondo secolo, Apuleius, Apologia 85. 
proporzionali alla sua presenza alla corte centrale. Così, si capisce la rappresentazione iconica del busto dell'imperatore fra nastri dorati sui codicilli, simbolo della diuina maiestas imperatoris:

"auctoritas and maiestas, derived from the language of the Roman state, did; it was chiefly by approaching the terms of Roman dignity for rulers that soveregnity was claimed. Great kings expressed their authority by claiming the title, powers and insignia of imperium itself within their own territories. This was intended to signify that they could act without reference to others, whether inside or outside the realm, and often that they had some right to rule other peoples and lands." ${ }^{30}$

Questi dittici erano dati all'ordo superioris, che legittimamente aveva il potere.

La consegna pubblica di questi documenti dell'amministrazione centrale, concessi alle dignitates, era simbolo dell'immagine legittima della summa potestas et auctoritas imperatoris che conferiva gli incarichi diretti del potere, era il potere assoluto e autocratico ratificato dall'ordo dignitatum e riflesso nella sua imago con una rassegna e accettazione pubblica. ${ }^{31} \mathrm{~A}$ sua volta l'imperatore è la rappresentazione dello Stato; egli è considerato come fonte necessaria di auctoritas. Intorno all'imperatore è originata un'attività che dipendeva dagli organismi periferici e dagli impiegati designati da lui stesso, che spedivano regolarmente rapporti all'amministrazione centrale primicerius notariorum -. Il procedimento burocratico iniziato da Diocleziano (295-305) è stato portato alle estreme conseguenze in un macchinoso e strutturato dispositivo amministrativo-burocratico negli ultimi dieci anni del quarto secolo, quando un' organizzata ed inamovibile gerarchia di dignitates gravitava intorno all'auctoritas imperatoris, sostenitore di un equilibrio perfetto degli ambiti civili e militari:

“'Ordine' indica il principio statico, 'conformità allo scopo' quello dinamico -un mezzo per perfezionare nel miglior modo

\footnotetext{
${ }^{30}$ A. Black, Political Thought in Europe, 1250-1450 (Cambridge 1992), 113-114. Cf. W.E. Gladstone, 'The Colour-Sense', The Nineteenth Century 2 (1877), 366-388; Bayer 1996, art.cit. (n.4), 15-16.

${ }^{31}$ Vegetius, De re militari 2.6. Cf. D.B. Capelle, 'Un Homiliaire de l'évèque Arien Maximin', Revue Bénédictine 34.1-4 (1922), 81-108; R.R.R. Smith, 'Late Antique Portraits in a Public Context. Honorific Statuary at Aphrodisias in Caria, AD 300-600', Journal of Roman Studies 89 (1999), 155 189; M. Stroll, Symbols as Power: the Papacy following the investiture contest (Leiden 1991).
} 
possibile l'imperfetto ordine terreno... All'immutabilità di principio dell'ordine corrispondono la stabilità dell'idea imperiale e la continuità della sua rappresentazione e della sua glorificazione nel cerimoniale e negli scritti dei retori.

L'imperatore creava una struttura gerarchica in cui inquadrava tutti gli altri sovrani come fratelli, figli o amici, considerava i loro doni come un riconoscimento del suo potere e i propri come segni del loro vassallaggio, per salvaguardare l'idea dell'universalità del proprio dominio." 32

A sua volta, il carattere ufficiale del documento è realizzato attraverso l'organizzazione gerarchica delle dignitates, che costituiscono il concetto di Imperium come entità dello Stato. La sua ricchezza iconografica ${ }^{33}$ e la preoccupazione per l'ornamento vanno al di là delle finalità del documento.

La Diuina Electio rappresenta e simboleggia l'aeternitas della potestas imperatoris, l'elezione evidente, intangibile e costante dell'imperatore da parte della divinità, ${ }^{34}$ cioè l'aspetto divino della sua dignità politica. L'immagine delle quattro stagioni - uernus, aestas, autumnus et hiems - lo confermano. Il simbolismo delle quattro stagioni è il divenire annuale del calendario astrale. L'imperatore occupa il posto del sole nella giustizia che è, a sua volta, il simbolo del Verbo di Dio per il quale e nel quale tutto è stato creato, illuminato e tutto è guidato. Esso simboleggia il posto centrale dell'ordine cosmico. La luce con il suo movimento circolare e le sue variazioni d'orbita determina non soltanto le notti e i giorni, ma anche le stagioni e i mesi, alla stessa maniera l'imperatore fissa le fasi dell'anno liturgico. Con la sua presenza egli determina i cicli naturali con il valore simbolico che essi trasmettono. Così si sottolinea il carattere ciclico dell'annus come pure l'imperfezione del suo carattere; in primo luogo perchè il suo movimento è continuo e infinito, in quanto appena concluso ricomincia nuovamente, anche se non arriva a rompere il cerchio del perpetuo inizio che non ha mai un termine e neanche un inizio: esso è segno di un'imperfezione radicale, quella in cui inciampano tutte le religioni cosmiche o naturali. In secondo luogo, il carattere ciclico è zoppo nel calcolo dei giorni e dei mesi. Da questo punto di vista l'annus è non stabilis sua post uestigia currit, espressione che spiega la

\footnotetext{
${ }^{32}$ Mertens 1999, op.cit. (n.3), 11-12; 14-15

${ }^{33}$ A.H.M. Jones, Cities of the Eastern Roman provinces (Oxford 1937), $492-493$.

${ }^{34} \mathrm{D}$. Mertens, 'Il potere temporale e il suo diritto', in Il pensiero politico Medievale (Bologna 1999), 85-93.
} 
sua radicale imperfezione e la sua incapacità di concludere. L'anno, sempre in fuga, ricorda l'eterna presenza del sole divino.

I numeri 4 e 24 sono i numeri che simboleggiano i ritmi fondamentali dei cicli naturali, le quattro stagioni e la divisione del giorno in 24 ore. $\mathrm{Si}$ profila così l'immagine del mondo che costituisce la caratteristica comune dell'antichità classica: la sfera dell'universo che effettua la sua rotazione liturgica attorno al centro immobile che la muove. Le quattro stagioni sono propriamente quelle che conferiscono all'immagine sul piano naturale il simbolismo cosmico dell'universalità, e sul piano teologico il significato di pienezza dell'ordine stabilito.

L'aspetto divino dell'auctoritas imperatoris è presente in entrambe le immagini attraverso l'armarium a forma di tabernacolo, nel caso della domus Augusti, che custodisce nei sacra scrinia la documentazione ufficiale che rappresenta le diverse cariche, civili e militari. Così gli elementi paralleli, da un punto di vista tematico, simbolico e strutturale, che fanno parte di entrambe immagini sono l'armarium e l'imago clipeata, cioè, l'aspetto antropomorfo e divino dell'auctoritas imperatoris. L'unione di tutti questi elementi sviluppa l'idea di equilibrio politico delle istituzioni, evidenziata attraverso i sacra scrinia che raccolgono in maniera frontale i codicilli messi insieme in una strutturata gerarchia lineare, parallelamente alla gerarchia delle istituzioni nel basso Impero e l'imago imperatoris fra $\mathrm{i}$ nastri d'oro. ${ }^{35}$ Tutto l'ordine e la disposizione gerarchica nell'illustrazione è riflesso univoco dell'ordine cosmico stabilito da Dio, il cui rappresentate terreno è l'imperatore. Tutto gravita intorno all'imago a cui deve rendere eternamente omaggio, gloria e lode. Il doppio significato di tali documenti con l'immagine dell'imperatore era politico e religioso: l'autorità indiscussa dell'imperatore era fondata e garantita dagli atti amministrativi o giuridici, fatti dai suoi delegati e legittimati dalla sua praesentia in absentia, mentre con un simbolismo di radice greca ed una riutilizzazione cristiana, il carattere sacro dell'imperatore è sottolineato dall'idea di eterno ritorno del suo potere, concezione ideologica dell'inviolabilità e continuità della sua auctoritas e delle virtù proprie di un bravo capo di Stato per l'integro compito delle sue cariche. ${ }^{36}$

\footnotetext{
${ }^{35}$ Le icone della parte orientale del testo sono stati considerati da una prospettiva bifocale e nella parte occidentale da una prospettiva tridimensionale, particolarità trovata nei mosaici di Ravenna, anni 421423. La collocazione lineare dei clipea rivela che la iconografia della $N D$ dall'inizio fu fatta sui rotoli di pergamena per essere risolta in codex.

${ }^{36}$ A. Zimmermann, Der Begriff der Repraesentatio im Mittelalter. Stelvertretung Symbolik Zeichen, Bild Miscellanea Mediaevalia 8 (Berlino-New York 1971).
} 


\section{Conclusione: la Notitia Dignitatum come paradigma di convinzioni iconografiche}

La progettata distribuzione bipartita e simmetrica della $N D$ fra precisi principi iconografici e strutturali, deriva da aspirazioni politiche di equilibrio dell'Impero di Teodosio, evidenziate mediante un linguaggio pittorico che raccoglie e chiarisce un testo accanto all'immagine, concepito secondo i principi di una precedente compilazione. Questa divisione iconografica e testuale fu progettata, all'inizio, come principio di un ordine politico e di accordo fra le due parti dell'Impero, come disposizioni testamentarie per un Impero diviso per la prima volta, ma regolato da normative simili sotto l'aspetto politico, burocratico, amministrativo e militare per futuri reggenti.

La distribuzione e iconografia sono articolati intorno a un fine preciso, quale la consonanza politica e la necessaria reciprocità tra le due parti dell'Impero. Le tendenze artistiche della fine del quarto e inizi del quinto secolo furono messe al servizio di una gerarchia, il cui fine fondamentale era l'espressione e la diffusione della politica teodosiana. Il vero fondamento sta negli affari politici dell'unità del potere centrale ${ }^{37}$ mantenuto da veloci ed uniformi cambiamenti del governo militare: all'inizio, fu il risultato del Breuiarium dell'Impero, però poi diventò, nei primi dieci anni del quinto secolo, un manifesto propagandistico di politica militare.

D'accordo con questi principi si possono capire le ragioni e lo scopo della $N D$, la quale sarebbe un notiziario annuale dell'Impero, ${ }^{38}$ associato ad una consuetudine burocratica consolidata e stabilita dal I secolo d.C., che aveva costituito normative precise e procedure metodologiche di controllo ed aggiornamento periodici, cui erano sottomessi documenti simili.

L'iconografia fu usata con uno stretto proposito politico: colori simbolici associati ad una semplicità delle icone assicurava, in assenza di un testo, la legittimità di un ordo dignitatum e di una potestas et ueneratio imperatoris come custode e sostenitore di un Impero diviso, il quale si legava giuridicamente al clero sotto il dominium imperatoris. L'arte come

\footnotetext{
${ }^{37}$ O. Seeck, ND. Accedunt Notitia urbis Constantinopolitanae et laterculi prouinciarum (Berlino 1876, reed.Francoforte 1962); G. Clemente, La ND (Cagliari 1968); J. Gaudemet, 'Recherches sur la législation du Bas-Empire', in Studi in onore di Gaetano Scherillo II (Milano 1972), 693-715; G. Nocera, 'Il Centralismo amministrativo del tardo impero', Accademia Romanistica Costantiniana, Atti VIII Convegno Internazionale (Perugia 1990), 599-621; P. Brennan, 'The User's Guide to the ND. The Case of the Dux Armeniae (ND.Or.38)', Antichthon 32 (1998), 34-49; A. Marrone, 'Un inedito di M.Rostovtzeff sulle cause della caduta dell'Impero romano', Historia 48 (1999), 254-256; M. Kulikowski, 'The $N D$ as a historical source', Historia 49 (2000), 358-377.

${ }^{38}$ S. Mazzarino, Trattato di storia romana Vol. II (Roma 1956), 446 n. 1.
} 
mezzo di propaganda politica è la spina dorsale della $N D$, la manifestazione artistica legata ai principi dell'ideologia della summa potestas dell'imperatore.

L'espressione politica del testo abbozzato negli ordini sociali testuali ed iconografici di fondamenti simbolici chiari è schematizzato attraverso le due immagini senza testo. ${ }^{39}$ Il suo significato testuale e contestuale è rappresentato attraverso l'indipendenza di tutti gli oggetti che fanno parte della iconografia. Le misure dei componenti nell'icona sono svolte attraverso un valore simbolico, il cui significato è equivalente e paragonabile al suo valore allegorico; la molteplicità delle scale iconografiche spinge la comprensione simbolica di ciascuno dei componenti fino al concepimento e sviluppo dell'icona, considerato come struttura prestabilita ed indivisibile, mentre le diverse strutture di ordinamento e sistemazione dello spazio nella figura confermano il suo significato metaforico. Da questo punto di vista la $N D$ è concepita come documento postumo di analoghe disposizioni dell'amministrazione che considerano i modelli della politica universale, sorretta dalla persona imperatoris. Testo ed immagine non possono avere rappresentazione più efficace, sia come traduzione visuale immediata del senso del testo in immagine, sia come percezione della loro equivalenza, sia per come si connotano i concetti stessi di testo ed immagine.

Firenze, Luglio 2002

\footnotetext{
${ }^{39} \mathrm{R}$. Grigg, 'Inconsistency and lassitude: the shield emblems of the $N D$ ', Joumal of Roman Studies 73 (1983), 132-142; idem, 'Illustrations and Text in the lost Codex Spirensis', Latomus 46 (1987), 204210.
} 